\title{
Quantitative Trait Loci and Candidate Genes Associated with Photoperiod Sensitivity in Lettuce (Lactuca spp.)
}

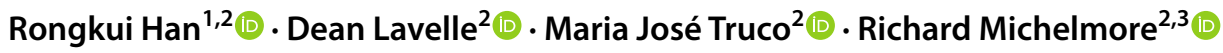

Received: 2 March 2021 / Accepted: 2 July 2021 / Published online: 10 July 2021

(c) The Author(s) 2021

\begin{abstract}
Key message A population of lettuce that segregated for photoperiod sensitivity was planted under long-day and short-day conditions. Genetic mapping revealed two distinct sets of QTLs controlling daylength-independent and photoperiod-sensitive flowering time.

Abstract The molecular mechanism of flowering time regulation in lettuce is of interest to both geneticists and breeders because of the extensive impact of this trait on agricultural production. Lettuce is a facultative long-day plant which changes in flowering time in response to photoperiod. Variations exist in both flowering time and the degree of photoperiod sensitivity among accessions of wild (Lactuca serriola) and cultivated (L. sativa) lettuce. An $\mathrm{F}_{6}$ population of 236 recombinant inbred lines (RILs) was previously developed from a cross between a late-flowering, photoperiod-sensitive L. serriola accession and an early-flowering, photoperiod-insensitive $L$. sativa accession. This population was planted under long-day (LD) and short-day (SD) conditions in a total of four field and screenhouse trials; the developmental phenotype was scored weekly in each trial. Using genotyping-by-sequencing (GBS) data of the RILs, quantitative trait loci (QTL) mapping revealed five flowering time QTLs that together explained more than $20 \%$ of the variation in flowering time under LD conditions. Using two independent statistical models to extract the photoperiod sensitivity phenotype from the LD and SD flowering time data, we identified an additional five QTLs that together explained more than $30 \%$ of the variation in photoperiod sensitivity in the population. Orthology and sequence analysis of genes within the nine QTLs revealed potential functional equivalents in the lettuce genome to the key regulators of flowering time and photoperiodism, FD and CONSTANS, respectively, in Arabidopsis.
\end{abstract}

\section{Introduction}

Flowering time is a complex phenotype affected by both the genetic makeup of the plant and environmental conditions (Srikanth and Schmid 2011). Environmental factors documented to influence flowering time include vernalization (Johanson et al. 2000; Gendall et al. 2001; Searle et al. 2006), ambient temperature (Balasubramanian et al. 2006; Reeves et al. 2007), daylength (Putterill et al. 1995; Searle

Communicated by Matthew N Nelson.

Richard Michelmore

rwmichelmore@ucdavis.edu

1 The Plant Biology Graduate Group, University of California, Davis 95616, USA

2 The Genome Center, University of California, Davis 95616, USA

3 Department of Plant Sciences, University of California, Davis 95616, USA and Coupland 2004), and light quality and intensity, due to their impact on accumulation of photosynthates (Cho et al. 2018). The control of floral initiation by daylength is termed photoperiodism. Daylength is a key indicator of seasonality; hence, it is an important environmental variable that determines the timing of a plant's transition to reproductive growth (Lang 1965). Arabidopsis thaliana is a facultative long-day (LD) plant, for which longer photoperiods accelerate flowering in an incremental fashion, while shorter photoperiods delay flowering but do not completely suppress it (Mouradov et al. 2002; Fornara et al. 2010). The current model for the molecular mechanism of the photoperiodic control of flowering time in Arabidopsis centers around a zinc finger transcription factor, CONSTANS $(C O)$. Arabidopsis alters flowering time as a result of differential accumulation of $\mathrm{CO}$ in its leaf tissues under different daylengths. A multi-layer regulatory network controls the quantity of $\mathrm{CO}$ by regulating the transcription level of the $\mathrm{CO}$ gene (Park et al. 1999; An et al. 2004; Imaizumi et al. 2005) and by coordinating ubiquitin-mediated post-translational 
degradation of its protein product in a phytochrome- and circadian-clock-dependent fashion (Jang et al. 2008). Given this regulatory network, $\mathrm{CO}$ accumulates more readily under LD conditions (Suárez-López et al. 2001). This subsequently promotes the expression of FLOWERING LOCUS T $(F T)$ in leaf tissues, from where the protein and mRNA encoded by $F T$ travel up the vascular system through phloem companion cells and transduce the floral initiation signal to the shoot apical meristem (Corbesier et al. 2007; Li et al. 2011).

Wild lettuce (Lactuca serriola), the wild progenitor of cultivated lettuce (L. sativa; Kesseli et al. 1991), is a facultative LD plant (Sukprakarn 1985). Cultivated lettuce also exhibits varying degrees of responsiveness to photoperiod, although its sensitivity is generally muted in comparison to its wild relative. Some cultivars (cvs.), such as the North American crisphead "Empire" and "Salinas," are less sensitive to photoperiod than others, such as the European butterheads "May King" and "Saffier," for which flowering time is significantly delayed as the daylength shortens (Waycott 1995). Lettuce is harvested for its vegetative tissues for consumption as a leafy vegetable in the western diet and for its enlarged vegetative stem in East Asian cuisines (Zhang et al. 2017). Floral initiation in lettuce is accompanied by the increase of bitter flavors (Ryder 1996), which drastically diminishes the culinary quality of the vegetable. As a result, delayed flowering is a major consideration in lettuce breeding to maximize harvestable yield (Thompson and Ryder 1961). Because the flowering time trait exhibits photoperiod dependency, it is also desirable to breed for lettuce with stable flowering time across different growing areas and daylength conditions. Understanding the genetics and molecular mechanism of the photoperiodic regulation of flowering time in lettuce can enhance the efficiency of breeding endeavors.

In the past decade, multiple genetic mapping and association studies have reported genetic loci controlling bolting (Lavelle 2009; Jenni et al. 2013; Mamo et al. 2019; Sthapit Kandel et al. 2020; Seki et al. 2020) and flowering time traits in lettuce (Hartman et al. 2012, 2013a, b; Kwon et al. 2013; Niroula 2017). A recent review on this topic reported a total of 64 quantitative trait loci (QTLs) associated with bolting and/or flowering time phenotypes in lettuce (Han et al. 2021a). Two QTLs on Chromosomes 2 and 7, qFLT2.1 and $q F L T 7.1$, have shown major effects on lettuce flowering time across multiple mapping populations. There have been fewer molecular studies on flowering time. No homolog or functional equivalent of $\mathrm{CO}$ has been identified in lettuce (Lavelle 2009; Abbott 2010; Han et al. 2021a). A clone of the lettuce ortholog of Arabidopsis FT, "LsFT" (Lsat_1_ v5_gn_2_17881) induced early flowering when ectopically expressed in transgenic Arabidopsis (Fukuda et al. 2011). Notably, qFLT2.1 co-locates with LsFT (Han et al. 2021a).

Past studies have only examined bolting and/or flowering time under single photoperiod conditions and the vast majority have been conducted under only LD conditions (Han et al. 2021a). The genetics underlying the response of lettuce to changing photoperiods has not been investigated. This study exploited available genetic and genomic resources in lettuce to distinguish the photoperiodic response phenotype from the daylength-independent flowering time phenotype. A population of $\mathrm{F}_{6}$ recombinant inbred lines (RILs) was previously developed from a cross between a late-flowering, photoperiod-sensitive accession of $L$. serriola, Armenian999, and an early-flowering, photoperiodinsensitive landrace line of L. sativa, PI251246 (Sandoya et al. 2020). The population was planted under LD and short-day (SD) conditions in multiple environments. We partitioned the flowering time phenotype into its two components: daylength-independent flowering time (FLT) and photoperiodic sensitivity (PPS). Separate QTLs were identified for FLT and for PPS. The QTLs for these two traits did not co-locate, indicating separate genetic determinants controlling FLT and PPS.

\section{Materials and methods}

\section{Plant material}

A population of $236 \mathrm{~F}_{6}$ RILs was previously developed by single-seed descent from a cross between the $L$. serriola accession Armenian999 and the L. sativa landrace line PI251246 (Sandoya et al. 2020). Armenian999 flowers slightly later than PI251246 under LD conditions and exhibits strong sensitivity to photoperiod. PI251246 exhibits low sensitivity to photoperiod and therefore flowering is not delayed as much as Armenian999 under SD conditions. This population is suitable for studying flowering phenotypes because both parents do not form heads, which facilitates accurate scoring of floral initiation.

\section{Nightbreak experiment}

The parental lines, Armenian999 and PI251246, were seeded on December 20th, 2019 and grown in a greenhouse at Davis, CA. Individual plants were grown in one-liter pots spaced one foot apart. Six plants of each line were randomized and grown on the same bench under $10 \mathrm{~h} / 14 \mathrm{~h}$ light/ dark cycles, while another six of each were randomized and grown in the same greenhouse on a separate bench with an additional nightbreak treatment. For the nightbreak treatment, the bench used for the treatment was surrounded by a non-light-permeable white plastic tarp; one hour of supplementary lighting was given from a high-pressure sodium growth light in the middle of the dark period every day. The time at which the first flower bud became visible was recorded for each plant. A photograph of one representative 
plant of each line from each treatment was taken on February 17th, 2019 using a Canon EOS 50D DSLR Camera.

\section{Growing conditions}

The 236 RILs, both parental lines, and two controls, $L$. sativa cv. Salinas and L. serriola accession US96UC23 were planted in complete randomized blocks at two separate field locations in Davis and Salinas, California in summer 2019 to characterize flowering time under LD conditions. The same lines were planted in a field in Holtville, California and in a screenhouse without supplementary lighting in Davis, California in winter 2019-2020 (November-March) to characterize flowering time under SD conditions. All plants were seeded into $16 \times 8$ cell trays and grown into seedlings in the UC Davis Vegetable Crop Greenhouses facility. At 4-6 weeks old, seedlings were transplanted into the field (LD-Davis, LD-Salinas, and SD-Holtville) or onegallon pots (SD-screenhouse). For field experiments, preplant N-P-K fertilizer, pre-emergence herbicide Balan DF, and post-emergence herbicide Kerb $50 \mathrm{~W}$ were applied to the fields at levels recommended by their respective labels. Details of the designs and plants of the experiments are shown in Table 1.

\section{Phenotyping}

The developmental stage of individual plants was scored weekly, starting two weeks after transplanting. Flowering time of an individual was quantified as the time at which the first flower bud became visible. The average flowering time was calculated for each plot. Flowering time in days was transformed into growing degree days (GDDs). The number of GDDs accumulated in a given day, $d$, is calculated using the following formula:

$\mathrm{GDD}_{d}=\mathrm{T}_{\text {mean, } \mathrm{d}}-\mathrm{T}_{\text {base }}$

This allowed for adjustment of the effect of temperature on flowering time, with $\mathrm{T}_{\text {base }}=5.5^{\circ} \mathrm{C}$ (Maynard 2014).
Flowering time in units of GDDs was used as the phenotype for QTL mapping. The average temperature of each day, $\mathrm{T}_{\text {mean, } d}$, was calculated in Celsius from hourly measurements collected from the National Centers for Environmental Information website (https://www.ncdc.noaa.gov/) for the UC Davis University Airport, CA (Station ID WBAN:00174, GPS coordinates $38.533^{\circ},-121.783^{\circ}$ ), Salinas Airport, CA (Station ID: WBAN:23233, GPS coordinates $36.6636^{\circ}$, $-121.6081^{\circ}$ ), and Imperial Co. Airport, CA (Station ID: WBAN:03144, GPS coordinates $32.83417^{\circ},-115.57861^{\circ}$ ) weather stations during the respective periods of the experiments. The weather stations in Davis, Salinas, and Imperial were $0.5 \mathrm{~km}, 7.1 \mathrm{~km}$, and $12.7 \mathrm{~km}$ from the experimental plots, respectively.

Phenotypic values of photoperiod sensitivity (PPS) of the RILs were estimated separately for each SD experiment. PPS was calculated by subtracting the mean LD flowering time, averaged between the LD-Davis and LD-Salinas experiments, from the SD flowering time in each SD experiment:

$\mathrm{PPS}_{\text {Holtville }}=\mathrm{SD}_{\text {Holtville }}-\mathrm{LD}_{\text {mean }}$

$\mathrm{PPS}_{\text {Screenhouse }}=\mathrm{SD}_{\text {Screenhouse }}-\mathrm{LD}_{\text {mean }}$

This method provides biologically interpretable quantifications of photoperiodic responses ("number of GDDs the flowering time was delayed due to SD conditions"). It has been used in studies of photoperiodism in multiple plant species, including rice (Maheswaran et al. 2000), wheat (Sourdille et al. 2000), soybean (Tasma et al. 2001), maize (Coles et al. 2010), and Arabidopsis (Méndez-Vigo et al. 2013).

A linear modeling method was used as an alternative measure of photoperiod sensitivity to provide independent validation for the quantification method described above. The SD phenotype data of all RILs from the Holtville field trial and the screenhouse trial were separately regressed with their genotype on the major LD flowering time QTL $q F L T 4.1$ using ordinary least square regression. The residuals of the regression were used as quantifications of photoperiod sensitivity:
Table 1 Design and planting information for four experiments in 2019

\begin{tabular}{lllllll}
\hline Photoperiod & Location & Growth condition & $\begin{array}{l}\text { Date of transplanting } \\
\text { (MM/DD/YYYY) }\end{array}$ & RILs & $\begin{array}{c}\text { Block } \\
\text { Plants } \\
\text { per } \\
\text { block }\end{array}$ \\
\hline $\begin{array}{l}\text { LD } \\
(\text { Avg } 14.39 \mathrm{~h})\end{array}$ & Davis, CA & Field & $05 / 08 / 2019$ & 236 & 2 & 6 \\
$\begin{array}{l}\text { LD } \\
(\text { Avg } 13.80 \mathrm{~h})\end{array}$ & Salinas, CA & Field & $06 / 05 / 2019$ & 236 & 2 & 8 \\
$\begin{array}{l}\text { SD } \\
(\text { Avg } 10.93 \mathrm{~h})\end{array}$ & Holtville, CA & Field & $11 / 07 / 2019$ & 236 & 2 & 8 \\
$\begin{array}{l}\text { SD } \\
(\text { Avg } 11.54 \mathrm{~h})\end{array}$ & Davis, CA & Screenhouse & $12 / 17 / 2019$ & 236 & 3 & 1 \\
\hline
\end{tabular}




$$
\begin{aligned}
& \mathrm{SD}_{\text {Holtville }}=\hat{\beta}_{\text {Holtville }} \times \mathrm{qFLT} 4.1_{\{0,1\}}+\mathrm{PPS}_{\text {Holtville }}^{\prime} \cdot \\
& \mathrm{SD}_{\text {Screenhouse }}=\hat{\beta}_{\text {Screenhouse }} \times \mathrm{qFLT} 4.1_{\{0,1\}}+\mathrm{PPS}_{\text {Screenhouse }}^{\prime} .
\end{aligned}
$$

This method of isolating the photoperiod sensitivity component of flowering time may produce less biologically interpretable measurements; nevertheless, it ensures numeric independence of the photoperiodic component and the LD flowering time component.

\section{Genotyping and QTL analysis}

Parental lines and RILs were subjected to genotyping-bysequencing using 100 bp paired-end Illumina HiSeq 4000 as described in (Han et al. 2021b). The parental lines, Armenian999 and PI251246, were also whole-genomeshotgun sequenced using $150 \mathrm{bp}$ and $100 \mathrm{bp}$ paired-end Illumina HiSeq4000 to $29 \times$ and $17 \times$ coverages, respectively. Sequencing results were mapped to version 8 of the lettuce reference assembly (Reyes-Chin-Wo et al. 2017; NCBI: GCA_002870075.2) using bwa-mem (Li 2013). High-confidence single nucleotide polymorphism (SNP) markers were identified using the software FreeBayes (Garrison and Marth 2012). A genetic map was constructed using the software LepMap3 (Rastas 2017). Detailed protocols for genotyping and genetic map construction are described in Han et al. (2021b). Heritability of the phenotypes was estimated using mixed effect modeling with R packages "synbreed" (Wimmer et al. 2012) and "sommer" (Covarrubias-Pazaran 2016), using location as fixed effect and marker-estimated genetic relationship as random effect. QTL analysis was performed using 2677 high-quality SNP markers, each representing one distinct genetic bin. Composite interval mapping was performed using the R package "qtl" (Broman et al. 2003) to determine QTL peaks, intervals, and effects. The significance threshold was determined using a 1000-iteration permutation test with $p<0.05$. The region within 1-log-of-odds (LOD) score of each locus with a local peak LOD score above the significant threshold was extracted as a QTL interval. The QTLs were named using an acronym of the phenotype ("FLT" for LD flowering time, "PPS" for photoperiod sensitivity), the chromosomal location of the QTL, and a number that reflects the order of discovery of the QTL after taking into account previously reported flowering time QTLs (Han et al. 2021a).

\section{Genomic analysis}

Lettuce orthologs of flowering-time genes in Arabidopsis were identified as follows: proteome of seven eudicot species, Arabidopsis thaliana, Solanum lycopersicum, Daucus carota, Cynara cardunculus, Helianthus annuus, L. serriola, and $L$. saliva, were clustered into orthology groups using software Orthofinder (Emms and Kelly 2015); lettuce genes within the same orthology groups as Arabidopsis flowering time genes were then extracted and located in the reference genome. Amino acid sequences within the orthology groups of Arabidopsis FD, Phytochromes and CONSTANS, were aligned using ClustalOmega (Madeira et al. 2019). The circadian expression profile of the putative orthologs was as described in Supplementary Table 3 of Han et al. (2021a). Single nucleotide variants, insertions, deletions, stop-loss variants, and stop-gain variants were identified between the parental lines using the software ANNOVAR (Wang et al. 2010).

\section{Results}

\section{LD phenotype and daylength-independent flowering time QTL}

The LD flowering time phenotype, measured in GDDs after planting (GAP), showed right-skewed Gaussian-like distribution in both the Salinas and Davis experiments (Fig. 1). The phenotypic distribution was truncated at the lower end in the Davis experiment because the earliest line flowered before the first occurrence of phenotyping. The difference between the earliest flowering event in the experiment and the time of first phenotyping was estimated to be less than a week, given the condition of the plants at the time of phenotyping. To avoid introducing bias, this error was not manually corrected. Phenotypic values collected from the two LD experiments showed a significant correlation with each other $\left(R^{2}=0.46, p<10^{-16}\right.$; Fig. 1). Plants in the Davis experiment showed overall earlier flowering. The mean flowering time in Davis was 120 GAPs earlier than in Salinas. Under LD conditions, the insensitive parent (PI251246) flowered at 828.6 GAP on average, while the sensitive parent (Armenian999) had an average flowering time of 1028.6 GAP (Table 2, Rows $1 \& 2$ ). Transgressive segregation of LD flowering time was observed on the right (later flowering) end of the phenotypic distribution in the Davis trial and on both ends of the distribution in the Salinas trial (Fig. 1).

The genotype of the RILs at 2677 polymorphic SNP sites were used to construct a genetic map. The map covered $1883 \mathrm{cM}$ in nine chromosomal linkage groups (LGs). The mean distance between each pair of adjacent markers was $0.7 \mathrm{cM}$. Three gaps between 5 and $7 \mathrm{cM}$ are present in this map located at 149.0-155.8 cM on linkage group 3, $62.8-68.1 \mathrm{cM}$ on linkage group 7, and 50.3-55.7 $\mathrm{cM}$ on linkage group 9. There was one gap of $10.4 \mathrm{cM}$ on linkage group 3. Four RILs were excluded from downstream analyses due to the large percentage of missing genotype data, 
Fig. 1 Correlation in long-day flowering time of the mapping population in two environments. The long-day flowering time of the Armenian999 ( $L$. serriola) $\times \mathrm{PI} 251246$ (L. sativa) $\mathrm{F}_{6}$ recombinant inbred line (RIL) population was scored in weekly intervals in two field experiments in Salinas and Davis, CA. The dot plot presents the phenotype of the same RILs in the two experiments (x-axis: Davis; y-axis: Salinas). Flowering time is expressed in growing degree days

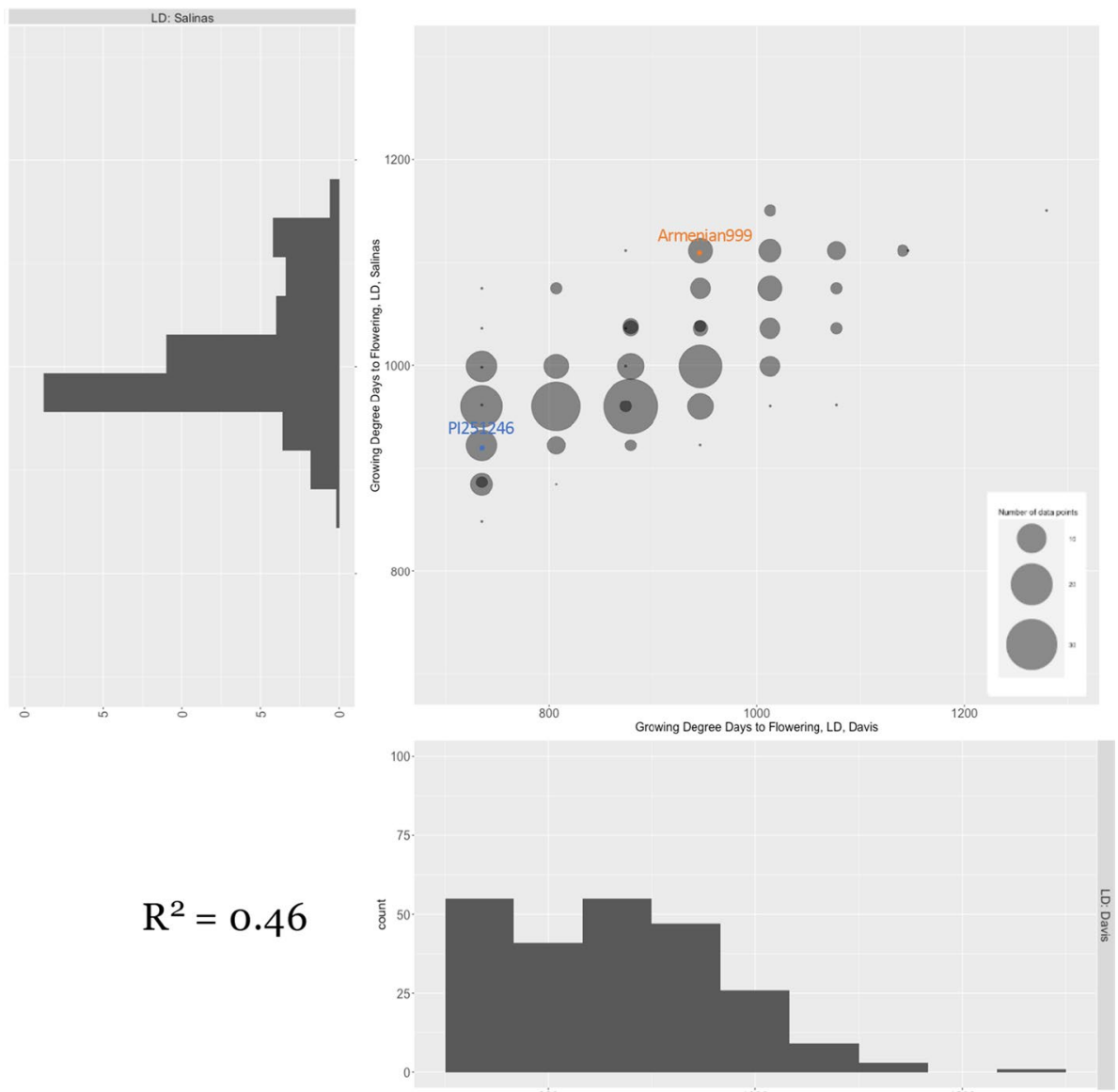

Table 2 Mean and range of values for flowering time, measured in growing degree days after planting, in a $L$. serriola $\times L$. sativa $\mathrm{F}_{6}$ RIL population and parents in two long-day (LD) experiments conducted in summer 2019 (Rows 1 \& 2), two short-day (SD) experi- ments conducted in winter 2019-2020 (Rows 3 \& 4), and photoperiodic response, derived from subtracting mean LD flowering time from experiment-level SD flowering time (Rows $5 \& 6$ ). SE indicates standard error

\begin{tabular}{|c|c|c|c|c|c|c|c|}
\hline \multirow[t]{2}{*}{ Phenotype } & \multirow[t]{2}{*}{ Location } & \multicolumn{2}{|l|}{ Parents } & \multicolumn{4}{|l|}{ RILs } \\
\hline & & PI251246 & Armenian999 & Min & $\operatorname{Max}$ & Mean & SE \\
\hline \multirow[t]{2}{*}{ LD flowering time } & Davis & 734.9 & 945.6 & 734.9 & 1279.2 & 873.6 & 107.8 \\
\hline & Salinas & 922.4 & 1111.5 & 848.6 & 1150.6 & 993.2 & 59.1 \\
\hline \multirow[t]{2}{*}{ SD flowering time } & Holtville & 961.4 & 1927.9 & 1011 & 1928 & 1571 & 253.5 \\
\hline & Screenhouse & 992.9 & 1556.5 & 952.1 & 1647.6 & 1230.2 & 159.2 \\
\hline \multirow[t]{2}{*}{ Photoperiodic response } & Holtville & 132.8 & 899.3 & 192.2 & 1080.3 & 639.9 & 253.5 \\
\hline & Screenhouse & 164.3 & 528.0 & 63.9 & 638.3 & 296.5 & 159.2 \\
\hline
\end{tabular}

resulting in a final set of 232 RILs for QTL mapping (Han et al. 2021b).

Broad sense heritability of flowering time under LD conditions was estimated to be 0.72 using a mixed effect model. Composite interval mapping revealed five significant QTLs for LD flowering time on LGs 4, 7, and 9. The individual QTLs accounted for $4.95-18.82 \%$ of the phenotypic variation (Fig. 2; Table 3). Two of these QTLs, qFLT4.1 and $q F L T 9.4$, were captured with QTL mapping that was re-performed using phenotypic data averaged across the two locations (Table 3).

QTL qFLT4.1 was detected in both experiments and had the highest LOD score among all LD flowering time QTLs. This QTL explained $12.05-18.73 \%$ of the variance in LD flowering time. The presence of the allele from the lateflowering parent Armenian999 on $q F L T 4.1$ delayed flowering. The allelic effect is reversed on qFLT9.2 and qFLT9.3, where the allele from PI251246 contributed to delayed 


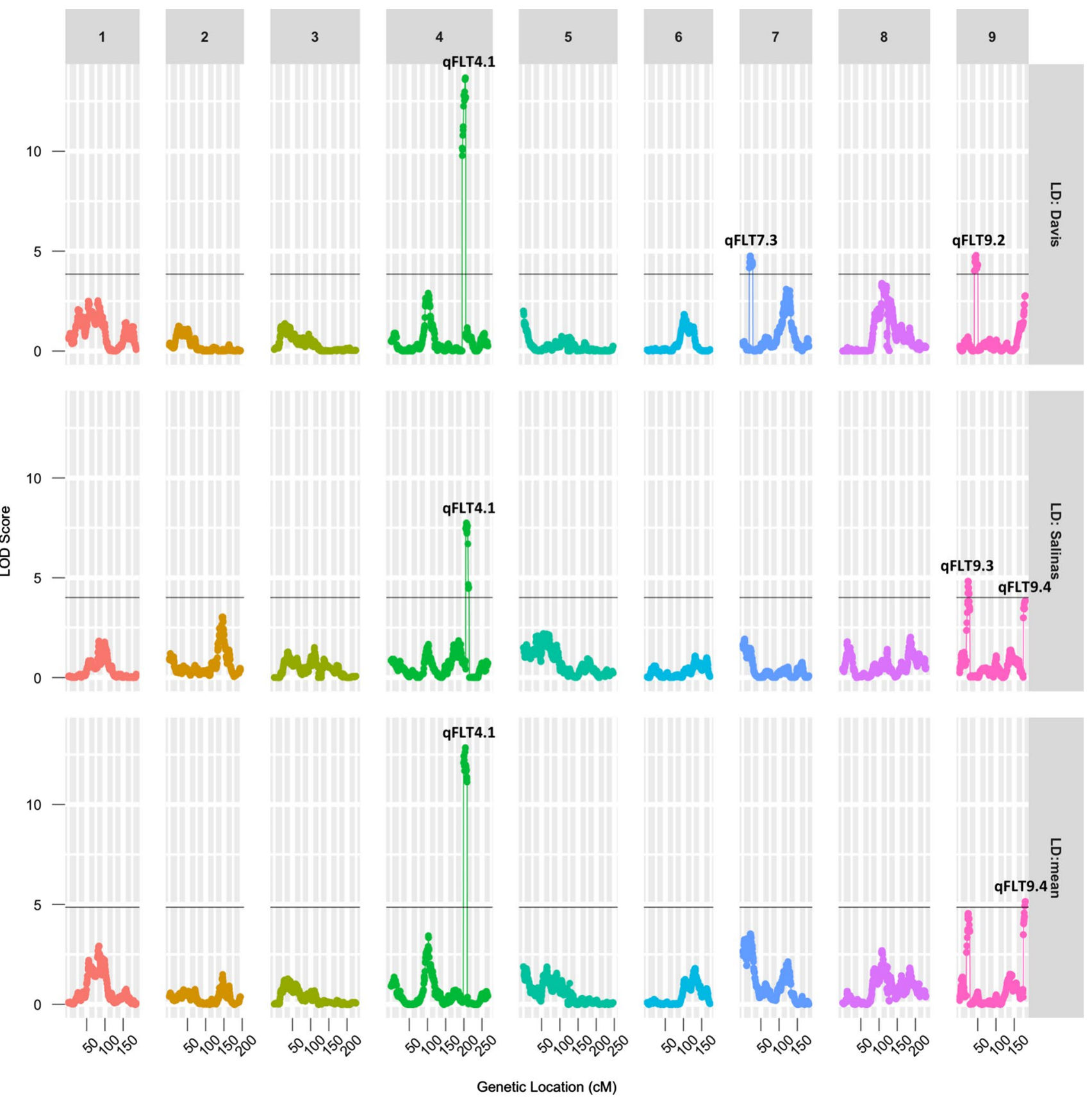

Fig. 2 Logarithm of odds (LOD) scores of markers for long-day flowering time, shown along the nine chromosomal linkage groups. The LOD threshold for significance $(p<0.05)$ calculated by 1000 permutations is shown as a black line

flowering. However, the effects of $q F L T 9.2$ and $q F L T 9.3$ were not significant when analyzing the mean phenotype across the two locations. No epistatic interactions were found between the QTLs.

\section{Nightbreak experiment}

The parents of the mapping population responded differently to one hour of light in the middle of the night (dark period) in an otherwise SD regime in the greenhouse (Fig. 3). The photoperiod-sensitive parent, Armenian999, flowered on average 78 days after planting (DAPs) in the control group and 57 DAPs in the nightbreak treatment; nightbreak significantly accelerated flowering by 21 days $\left(p<2 \times 10^{-16}\right)$. In contrast, the insensitive parent, PI251246, flowered on average 43 DAPs in the control group and 34.25 DAPs in the nightbreak group; nightbreak only accelerated flowering by 8.75 days and was not found to be significant $(p=0.12)$. 
Table 3 A total of five QTLs, qFLT4.1, 7.3, 9.2, 9.3 , and 9.4 , were detected for LD flowering time in an Armenian999 $\times$ PI $251246 \mathrm{~F}_{6}$ RIL population evaluated in two field experiments in summer 2019

Fig. 3 Contrasting responses of the parental lines to the nightbreak treatment. The plant on the left in each panel was grown for 61 days under $10 \mathrm{~h} / 14 \mathrm{~h}$ light/dark cycles in a greenhouse; the plant on the right in each panel was grown in the same greenhouse with an additional 1-h nightbreak treatment implemented in the middle of the dark period. a Photoperiod sensitive parent, Armenian999. b Photoperiod insensitive parent, PI251246

\begin{tabular}{|c|c|c|c|c|c|c|c|}
\hline Location & QTL & $\mathrm{Chr}$ & Marker closed to peak & Interval (cM) & $\mathrm{LOD}^{\ddagger}$ & $\mathrm{PVE}^{*}$ & Allele $^{\dagger}$ \\
\hline \multirow[t]{3}{*}{ Davis } & $q F L T 4.1$ & 4 & Lsat & 195. & 14.06 & 18.73 & A \\
\hline & $q F L T 7.3$ & 7 & Lsat_1_v8_lg_7.30591573_30591595 & $22.0-30.5$ & 4.56 & 4.95 & A \\
\hline & qFLT9.2 & 9 & Lsat_1_v8_lg_9.48974335_48974387 & $40.4-55.6$ & 5.81 & 6.13 & $\mathrm{P}$ \\
\hline \multirow[t]{3}{*}{ Salinas } & $q F L T 4.1$ & 4 & Lsat_1_v8_lg_4.294973197_295012418 & 198.0-209.0 & 7.98 & 12.05 & A \\
\hline & qFLT9.3 & 9 & Lsat_1_v8_lg_9.29591529_30108481 & $22-26.6$ & 4.67 & 7.35 & $\mathrm{P}$ \\
\hline & $q F L T 9.4$ & 9 & Lsat_1_v8_lg_9.200741908_204015989 & $175.2-180.8$ & 4.67 & 6.65 & A \\
\hline \multirow[t]{2}{*}{ Mean } & $q F L T 4.1$ & 4 & Lsat_1_v8_lg_4.283169022_283617815 & 198.0-206.0 & 13.01 & 18.9 & A \\
\hline & qFLT9.4 & 9 & Lsat_1_v8_lg_9.200088730_204243350 & 176.9-180.8 & 5.06 & 6.51 & A \\
\hline
\end{tabular}

†: Log of odds

*: Percent variance explained

$\dagger$ : The parental allele that increased the trait value. "A" represents Armenian999 and "P" represents PI251246. The same abbreviations were used in all other tables

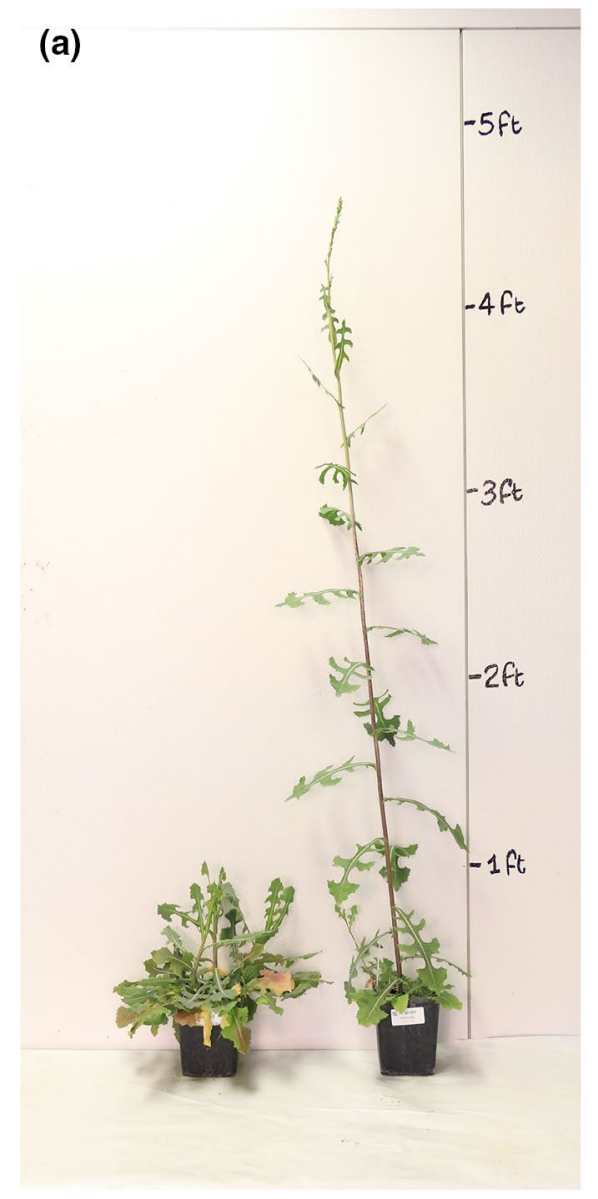

\section{(b)}

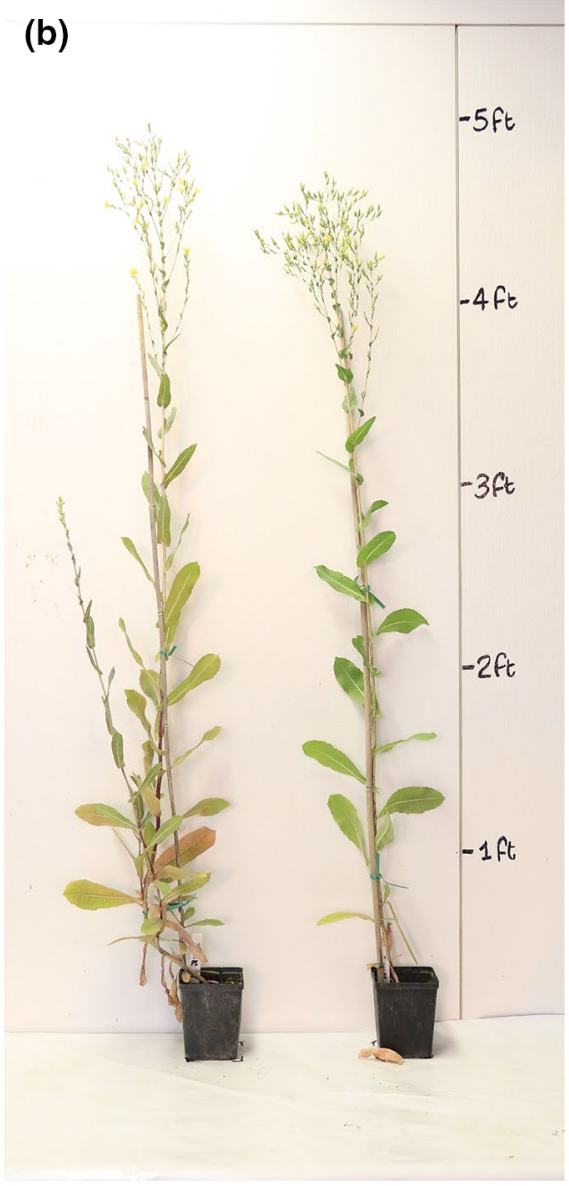

\section{Partitioning of photoperiod sensitivity component of the flowering time phenotype}

The genetics of sensitivity to daylength was examined by growing the same RIL population under SD conditions in the field and in a screenhouse with no supplementary lighting. Comparison of plot-level SD and LD flowering time data (in GAP) revealed that, under short photoperiod conditions, flowering was barely delayed in the insensitive parent PI251246 $(p=0.11)$ but significantly delayed in Armenian999 $\left(p=2.56 \times 10^{-6}\right.$; Fig. 4). On a population level, the LD flowering time of a RIL is a poor predictor of its SD flowering time; the average pairwise coefficient of determination $\left(R^{2}\right)$ is 0.33 for any pair of LD-SD experiments. This indicates that, in addition to daylength-independent flowering time regulation, there are separate genetic 


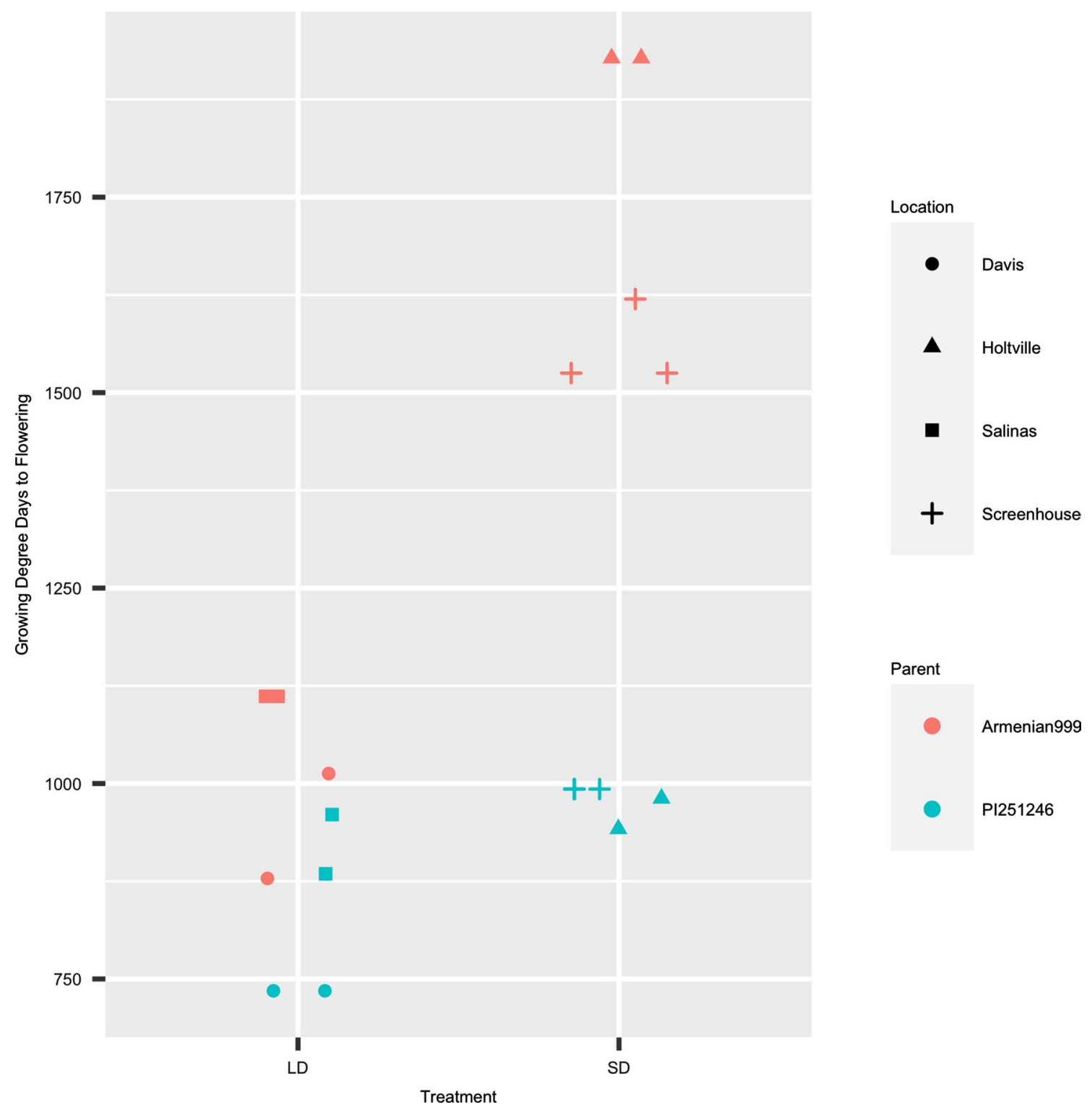

Fig. 4 Flowering time (in growing degree days) of the parental lines, Armenian999 (L. serriola) and PI251246 (L. sativa) in both long-day (Salinas and Davis) and both short-day (Holtville and screenhouse)

mechanisms for photoperiodic regulation of reproductive growth. In addition, the SD flowering time phenotype exhibited higher location-sensitivity than the LD flowering time phenotype. The SD flowering time phenotype had lower, although still significant, correlation between experiments $\left(R^{2}=0.38, p<10^{-16}\right)$, with the mean SD flowering time 339.4 GAPs later in the Holtville experiment in the Imperial Valley than in the screenhouse experiment at Davis (Table 2, Rows $3 \&$ 4; Fig. 5). The phenotype followed a right-skewed Gaussian-like distribution in the screenhouse experiment; in the Holtville field experiment, the phenotype showed clear bimodal distribution, with two peaks at 1300 and 1800 GDDs (Fig. 5). The bimodal distribution of experiments. Armenian999 exhibited a strong photoperiodic response in flowering time, while PI251246 was insensitive

the phenotype in the Holtville experiment is consistent with the presence of at least one major genetic locus that control daylength sensitivity. Broad sense heritability of flowering time under SD conditions was estimated to be 0.89 using a mixed effect model.

Phenotypic values of PPS of the RILs in each SD experiment were calculated by subtracting the mean LD flowering time from the SD flowering time in each SD experiment. Flowering of the sensitive parent, Armenian999, was significantly delayed as it flowered 899.3 (Holtville) and 528.0 (screenhouse) GDDs later in SD conditions than in LD conditions. In contrast, flowering of the insensitive parent PI251246 was delayed much less as it flowered 132.8 
Fig. 5 Phenotypic distributions of short-day flowering time of the Armenian999 ( $L$. serriola) $\times \mathrm{PI} 251246$ (L. sativa) $\mathrm{F}_{6}$ RIL population in two experiments conducted in an experimental field in Holtville, CA and a screenhouse in Davis, CA under short-day winter conditions. The dot plot presents the phenotype of the same RILs in the two experiments (x-axis: Holtville; y-axis: screenhouse). Flowering time is expressed in growing degree days

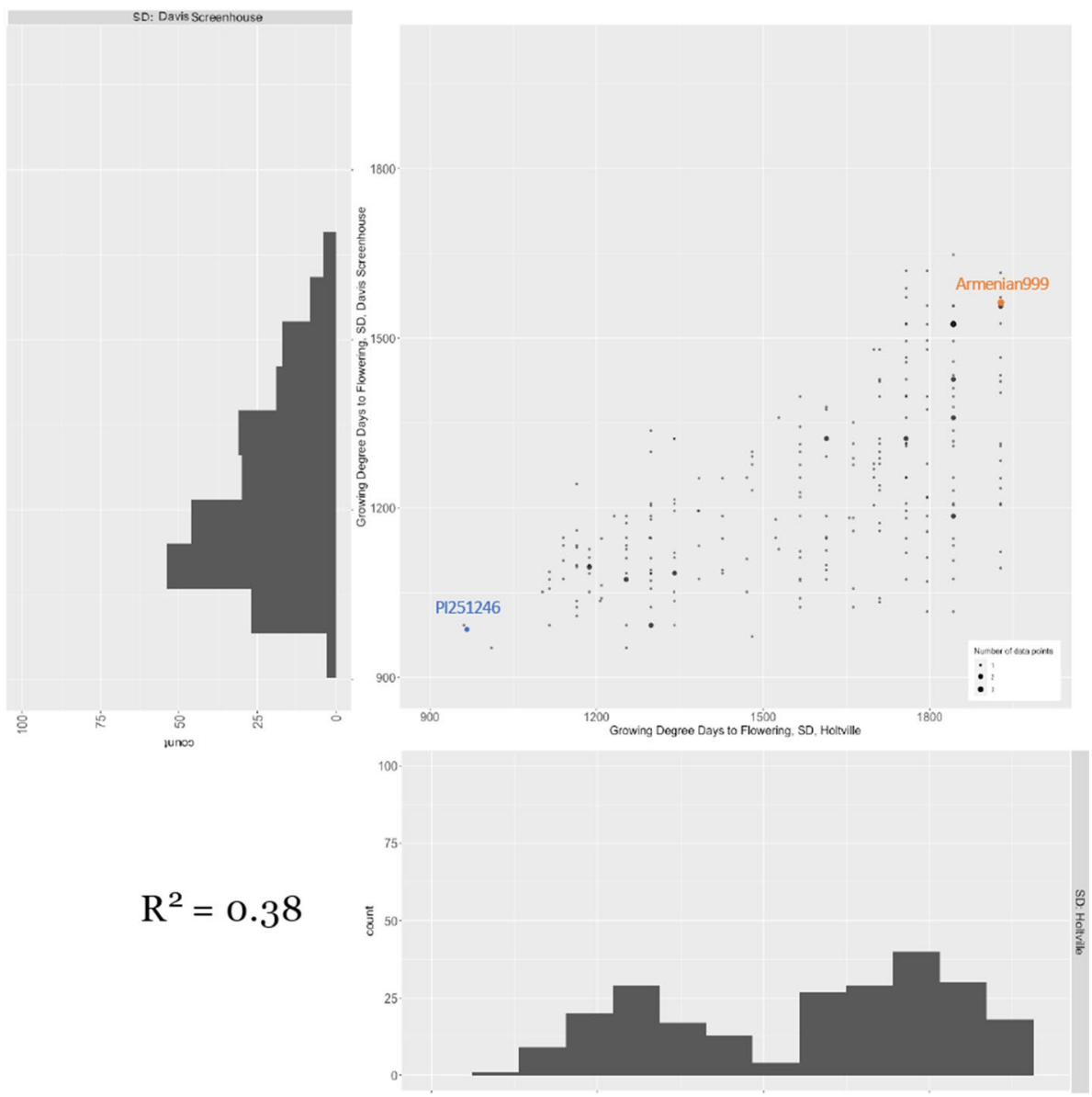

the Holtville experiment and $33.45 \%$ in the screenhouse experiment.

The result of the QTL analysis was confirmed using an independent data analysis protocol that calculated PPS by regressing SD phenotype using the genotype of the major LD flowering time QTLs qFLT4.1 as fixed effect (Fig. 7). The results of the two independent analyses strongly agree with each other $\left(p<10^{-16}\right.$; Supp. Figure 1). The three most consistent and largest-effect PPS QLTs, qPPS1.1, 2.1, and 4.1, were discovered using both methods (Supp. Table 1a). QTL analysis was also performed using just the SD flowering time data. This revealed the same three QTLs (qPPS1.1, 2.1 and 4.1) plus qFLT4.1, which corresponds to the locus controlling flowering time under both LD and SD conditions (Supp. Table 1b; Supp. Figure 2). The comparison of LD, PPS, and SD QTLs is presented in Supp. Figure 3.

\section{Candidate genes}

All of the detected FLT and PPS QTLs, except for qPPS1.2, contain genes that are orthologous to genes with flowering time related functions in Arabidopsis. Forty-five of these genes harbor non-synonymous variants between the parental lines within their coding sequences explained $44.5 \%$ of variance in photoperiod sensitivity in 
Table 4 A total of five QTLs were detected for photoperiod sensitivity in a $L$. serriola $\times$ L. sativa $\mathrm{F}_{6}$ RIL population evaluated in two long-day experiments and two short-day experiments in summer 2019 and winter 2019-2020

\begin{tabular}{|c|c|c|c|c|c|c|c|}
\hline Location & QTL & $\mathrm{Chr}$ & Marker closed to peak & Interval (cM) & LOD & PVE & Allele* \\
\hline \multirow[t]{3}{*}{ Holtville } & $q P P S 2.1$ & 2 & Lsat_1_v8_lg_2.171422510_171778602 & $132.0-135.8$ & 19.77 & 28.98 & A \\
\hline & $q P P S 4.1$ & 4 & Lsat_1_v8_lg_4.33492617_34012680 & $12.4-20.6$ & 6.39 & 10.27 & A \\
\hline & qPPS4.2 & 4 & Lsat_1_v8_lg_4.192761242_193410678 & $145.0-151.5$ & 5.50 & 9.13 & A \\
\hline \multirow[t]{3}{*}{ Screenhouse } & qPPS1.1 & 1 & Lsat_1_v8_lg_1.46475164_46488701 & $49-55.4$ & 12.84 & 20.07 & A \\
\hline & $q P P S 1.2$ & 1 & Lsat_1_v8_lg_1.189492341_201862756 & $175.0-182.0$ & 4.51 & 6.05 & A \\
\hline & $q P P S 2.1$ & 2 & Lsat_1_v8_lg_2.170127374_170849299 & $128.6-138.8$ & 5.93 & 7.39 & A \\
\hline \multirow[t]{4}{*}{ Mean } & qPPS1.1 & 1 & Lsat_1_v8_lg_1.50417917_50417917 & $49.0-60.0$ & 5.71 & 6.82 & A \\
\hline & $q P P S 1.2$ & 1 & Lsat_1_v8_lg_1.190901262_2027901202 & $176.0-181.1$ & 4.41 & 5.59 & A \\
\hline & $q P P S 2.1$ & 2 & Lsat_1_v8_lg_2.170127374_170849299 & $132.0-135.8$ & 17.28 & 26.65 & A \\
\hline & $q P P S 4.1$ & 4 & Lsat_1_v8_lg_4.43189585_43189629 & $22.0-33.0$ & 5.69 & 8.64 & A \\
\hline
\end{tabular}

*: The parental allele that increased the trait value

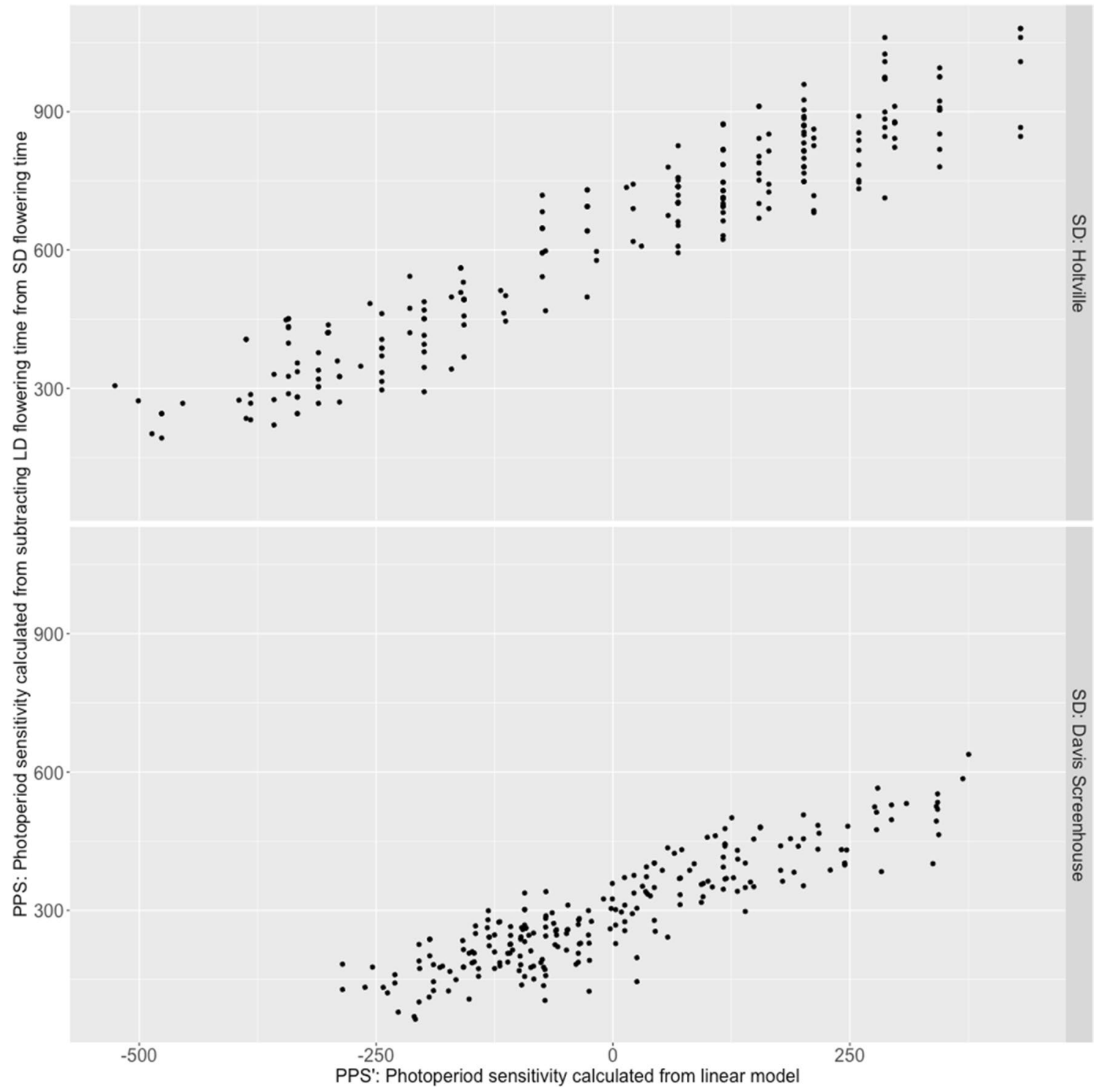

Fig. 6 Strong correlation between results of two methods of quantifying photoperiod sensitivity (PPS) 


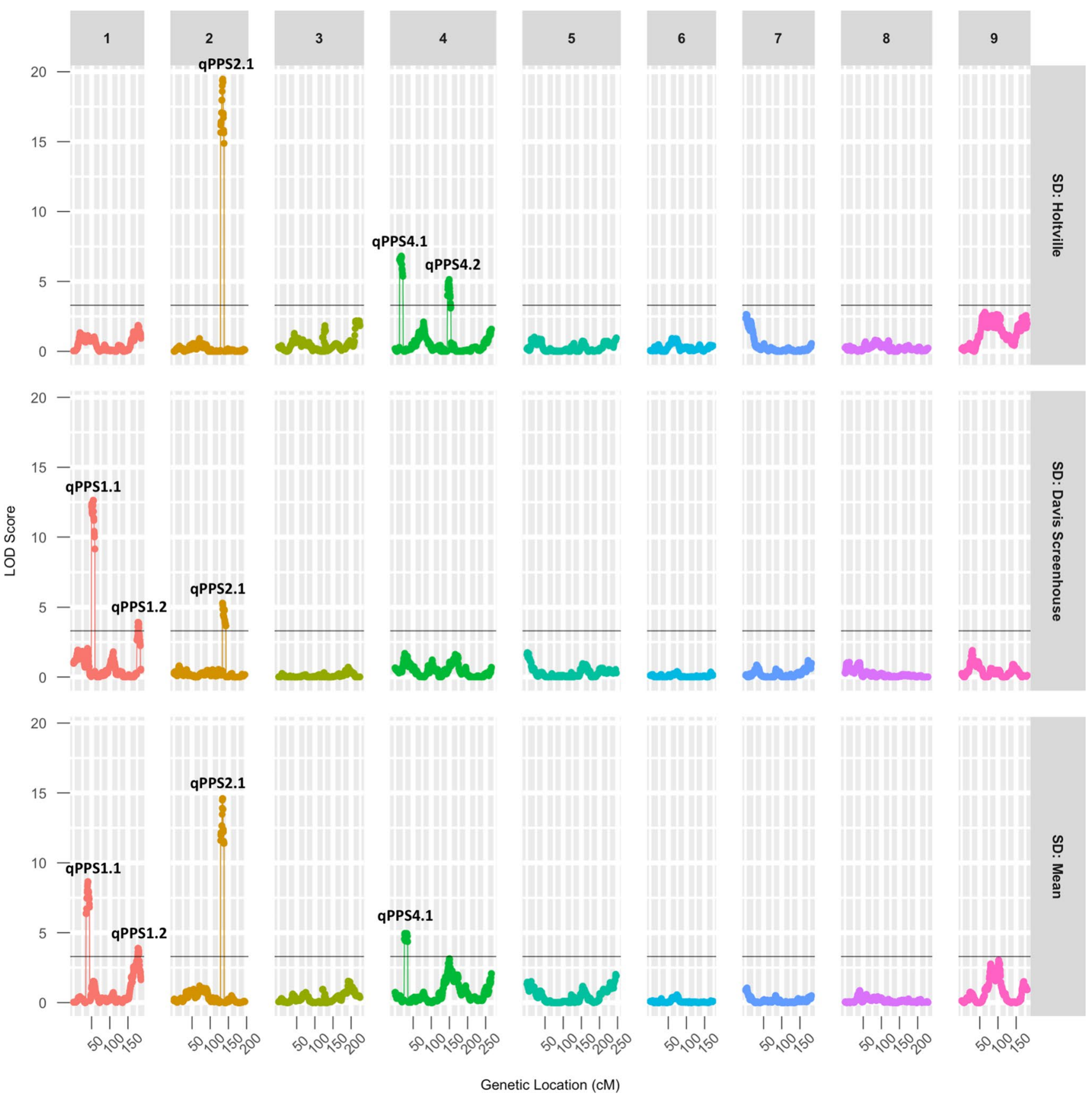

Fig. 7 LOD scores of markers for photoperiod sensitivity shown along the nine chromosomal linkage groups. The LOD threshold for significance $(p<0.05)$ calculated by 1000 permutations is shown as a black line

(Supplementary Table 2). Six of these genes show a rhythmically oscillating expression pattern during a day-night cycle in vegetative lettuce leaf tissues (Supplementary Table 2; Higashi et al. 2016). Among them, three have orthologs that function in the photoperiod pathway in Arabidopsis, including Lsat_1_v5_gn_2_86121 (orthologous to CO; Putterill et al. 1995) in qPPS2.1, Lsat_1_v5_ gn_4_19021 (orthologous to PFT1; Iñigo et al. 2012) in
qPPS4.1, and Lsat_1_v5_gn_4_108141 (orthologous to $C D F 1$; Imaizumi et al. 2005) in qPPS4.2. The 48-h expression profile of these three genes in vegetative lettuce leaf tissue grown under $12 \mathrm{~h} / 12 \mathrm{~h}$ light/dark cycles is shown in Fig. 8. A detailed summary of all non-synonymous sequence variants within candidate genes for all QTLs are listed in Supplementary Table 2. 
Fig. 8 Forty-eight-hour expression profiles of three candidate genes in QTLs $q P P S 2.1$ (Lsat_1_v5_gn_2_86121, orthologous to AtCO), qPPS4.1 (Lsat_1_v5_gn_4_19021, orthologous to AtPFT1), and PPPS4.2 (Lsat_1_v5_ gn_4_108141, orthologous to $A t C D F)$ in vegetative lettuce leaf tissues. Time-series RNAseq data was obtained from the $12 \mathrm{~h}$ light $/ 12 \mathrm{~h}$ dark experiment described in Higashi et al. (2016). Expression was quantified in reads per million mapped reads

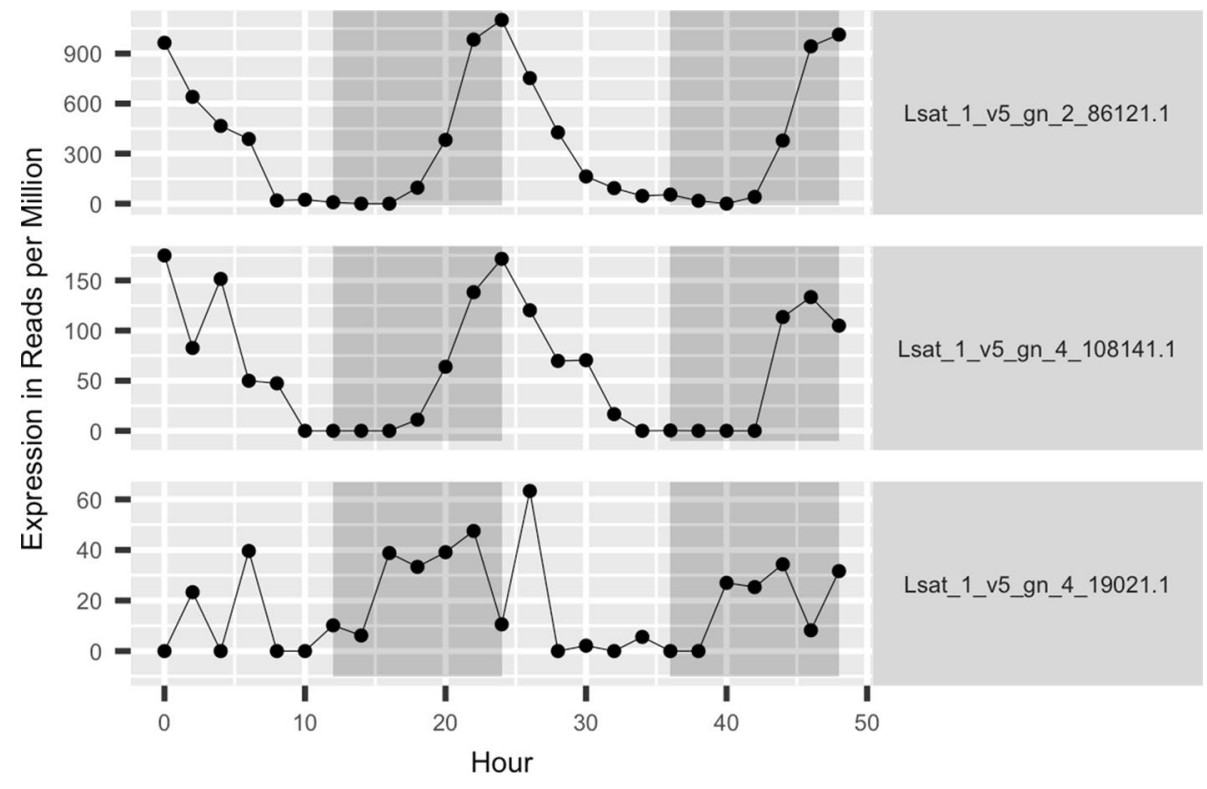

\section{Discussion}

In this study, a lettuce RIL population that segregated for both daylength-independent flowering time and photoperiod sensitivity was grown under multiple daylength conditions. QTL analysis revealed that these two phenotypes were determined by different loci. Both flowering time and photoperiod sensitivity showed high heritability. Photoperiod sensitivity exhibited higher variation across different environments than daylength-independent flowering time. We identified five QTLs determining daylength-independent flowering time and five QTLs determining photoperiod sensitivity. These QTLs together do not explain as much phenotypic variation as the narrow-sense heritability of the traits. Marker density is unlikely to be the cause of this discrepancy. The genetic map used for the analysis had 2677 markers with a mean distance between adjacent markers of $0.7 \mathrm{cM}$. Only one gap (of $11 \mathrm{cM}$ ) was larger than $7 \mathrm{cM}$. Even at this marker spacing, a large effect QTL should have been detectable. Another possible reason for such a discrepancy is the segregation of several additional minor effect QTLs for photoperiod sensitivity, which were not detected as significant; numerous QTLs for flowering have been reported for lettuce (Han et al. 2021b).

There was no overlap between the FLT and PPS QTLs, suggesting separate mechanisms. The largest QTL determining photoperiod sensitivity differed in the two SD environments; qPPS2.1 was the most significant in the Holtville experiment, while $q P P S 1.1$ was the most significant in the Davis Screenhouse experiment that on average had a longer photoperiod by $30 \mathrm{~min}$. This parallels experiments with Arabidopsis, in which photoperiodic regulation of flowering time differed between lab and field conditions (Song et al.
2018). This is consistent with the complex, environmentally sensitive regulatory network that determines the photoperiodic responses in flowering plants.

The largest effect flowering time QTL was $q F L T 4.1$. This overlapped with a bolting time QTL, $q B L T 4.1$, discovered in a $F_{6}$ RIL population developed from a cross between a Batavia variety "Reine des Glaces" and a Latin variety "Eruption" (Mamo et al. 2019) and encompasses 280 lettuce gene models. Among them is an ortholog to Arabidopsis $F D$ (Abe et al. 2005), Lsat_1_v5_gn_4_145080, and an ortholog to Arabidopsis FLOWERING LOCUS K (FLK; Lim et al. 2004), Lsat_1_v5_gn_4_138841. Both gene models carry non-synonymous variants between the coding sequences of the two parental lines (Supp. Table 2). The expression of Lsat_1_v5_gn_4_145080 in the lettuce apical meristem was quantified in Chen et al. (2018); in their study, the expression of Lsat_1_v5_gn_4_145080 reached a global maximum during the transition between vegetative growth and reproductive growth and decreased after the apical meristem committed to floral development. This is consistent with the known function of $F D$ in Arabidopsis where it complexes with FT in the apical meristem to promote the floral transition (Abe et al. 2005). Armenian differs from PI251246 at five amino acid residues between positions 100 and 240 . Although this region is not conserved between lettuce and Arabidopsis FD, this region is conserved across orthologs of species within the Compositae family, including lettuce, artichoke, and sunflower (Suppl. Table 4a). This does not rule out Lsat_1_v5_gn_4_145080 as a candidate for determining the phenotype of $q F L T 4.1$.

In addition to $q F L T 4.1$, two other flowering time QTLs collocate with the previously reported QTLs, qFLT7.4 and $q F L T 9.2$. qFLT7.4 was first discovered in a Salinas 
(crisphead) $\times$ PI171674 (romaine) $\mathrm{F}_{2}$ mapping population (Sandoya et al. 2020). This mapping population does not share parents with the population used in our experiment. $q F L T 9.2$ partially collocated with a peak identified in a genome-wide association study on lettuce that investigated variations in developmental rate (Sthapit Kandel et al. 2020). There are several flowering time related orthologs within these two QTLs; however, none have non-synonymous substitutions within their coding regions. Expression level polymorphism data are not available for these lines.

Many genes encoding putative photoperiodism-related components were identified within the two largest-effect PPS QTLs. A phytochrome encoding gene, Lsat_1_v5_ gn_1_41100, is located within qPPS1.1. Phytochromes constitute important components of the photoperiod pathway of flowering regulation in Arabidopsis (Legris et al. 2019). The phyB-phyC heterodimer is important for inhibiting flowering in non-inductive photoperiods (Monte et al. 2003; Sánchez-Lamas et al. 2016). Armenian differs from PI251246 at amino acid number 507; however, this position is not highly conserved between putative phytochrome genes in eudicot species (Suppl. Table 4b).

The other large-effect PPS QTL, $q$ PPS2.1, collocates with a bolting time QTL, $q S T L 2.2$, discovered in a $\mathrm{F}_{7}$ RIL population developed from a cross between two crisphead cvs. "Emperor" and "El Dorado" (Jenni et al. 2013). qPPS2.1 includes a CO-like ortholog, Lsat_1_v5_gn_2_86121. The expression level of $C O$ is circadianly entrained in Arabidopsis, resulting in a 24-h-phase oscillation of its transcription in vegetative tissues (Suárez-López et al. 2001). Lsat_1_ v5_gn_2_86121 also shows a circadian expression pattern in vegetative lettuce leaves (Higashi et al. 2016; Fig. 8). The first non-synonymous variant between Armenian999 and PI251246 is in the $64^{\text {th }}$ base pair of exon 1 (Supplementary Table 2, row 143). This substitution changes a threonine in Armenian999 and the genome reference cultivar, Salinas, to proline in PI251246 at position 22 of the encoded protein. The threonine at position 22 is in the middle of the first of the two zinc fingers of $\mathrm{CO}$ and is conserved between the daylength sensitive genotypes of lettuce and Arabidopsis (Putterill et al. 1995; Suppl. Table 4c). Of the 17 CO-like (COL) genes in the Arabidopsis genome, only two of them, COL5 and $C O L 9$, have thus far been shown to function in photoperiod control of flowering time (Cheng and Wang 2005; Hassidim et al. 2009). There are at least six COL paralogs in the lettuce genome (Han et al. 2021a); however, the functional ortholog of $C O$ had not been previously identified in lettuce, despite molecular and bioinformatic efforts (Abbott 2010). Our data indicates that Lsat_1_v5_gn_2_86121 is the functional homotholog of $C O$ in lettuce.

In summary, an interspecific Lactuca RIL population that segregates for both daylength-independent flowering time and photoperiod sensitivity provided an effective platform for studying the genetic mechanism of photoperiodic control of flowering time in lettuce. Our study showed that the photoperiodic regulation of flowering time in lettuce is distinct from genes determining daylength-independent flowering time under inductive daylength conditions. This study also revealed lettuce genes that are candidates for functional orthologs of $F D$ and $C O$, key flowering time and photoperiodism regulators in Arabidopsis. The identification of the genes that fulfill these roles in lettuce has been confounded in the past due to multiple paralogs in the duplicated lettuce genome. This study provides the foundation for future experiments focused on the functional validation of these candidate genes using genome editing and transgenic complementation.

Supplementary Information The online version contains supplementary material available at https://doi.org/10.1007/s00122-021-03908-w.

Acknowledgements We thank F. Banuelos-Gonzalez for field assistance, J. Emerson for greenhouse and field assistance, E. Georgian for editorial assistance, E. Kang for screenhouse and greenhouse assistance, and A. Vargas for DNA and GBS library construction.

Author Contribution statement RM, DL, MJT, and RH conceived the experiment. RH designed and conducted the field, screenhouse, and greenhouse experiments, performed the data analysis and genetic mapping, and drafted the paper. MJT and DL developed the mapping population, genotyped by sequencing and assisted in implementing the experiments. All authors contributed to writing the paper.

Funding This research was funded by an NSF Graduate Research Fellowship to RH and a USDA NIFA Specialty Crop Research Initiative (SCRI) Grant \# 2015-51181-24283 to RWM.

Data Availability GBS data of the RILs and WGS data of the parents are available on the NCBI SRA database under BioProjects PRJNA642889, PRJNA510128, and PRJNA478460. All raw phenotype data are available in Supplementary Table 3.

\section{Declarations}

Conflict of Interest The authors declare that the research was conducted in the absence of any commercial or financial relationships that could be construed as a potential conflict of interest.

Open Access This article is licensed under a Creative Commons Attribution 4.0 International License, which permits use, sharing, adaptation, distribution and reproduction in any medium or format, as long as you give appropriate credit to the original author(s) and the source, provide a link to the Creative Commons licence, and indicate if changes were made. The images or other third party material in this article are included in the article's Creative Commons licence, unless indicated otherwise in a credit line to the material. If material is not included in the article's Creative Commons licence and your intended use is not permitted by statutory regulation or exceeds the permitted use, you will need to obtain permission directly from the copyright holder. To view a copy of this licence, visit http://creativecommons.org/licenses/by/4.0/. 


\section{References}

Abbott Aa (2010) The isolation of flowering time genes from lettuce to enable the manipulation of bolting time. The University of Warwick

Abe M, Kobayashi Y, Yamamoto S et al (2005) FD, a bZIP protein mediating signals from the floral pathway integrator FT at the shoot apex. Science 309(5737):1052-1056. https://doi.org/10. $1126 /$ science. 1115983

An H, Roussot C, Suárez-López P et al (2004) CONSTANS acts in the phloem to regulate a systemic signal that induces photoperiodic flowering of Arabidopsis. Development 131:3615-3626

Balasubramanian S, Sureshkumar S, Lempe J, Weigel D (2006) Potent induction of arabidopsis thaliana flowering by Elevated growth temperature. PLOS Genet 2:e106

Broman KW, Wu H, Sen Ś, Churchill GA (2003) R/qtl: QTL mapping in experimental crosses. Bioinform 19:889-890. https://doi.org/ 10.1093/bioinformatics/btg112

Chen Z, Zhao W, Ge D et al (2018) LCM-seq reveals the crucial role of LsSOC1 in heat-promoted bolting of lettuce (Lactuca sativa L.). Plant J 95:516-528. https://doi.org/10.1111/tpj.13968

Cheng X-F, Wang Z-Y (2005) Overexpression of COL9, a CONSTANS-LIKE gene, delays flowering by reducing expression of $\mathrm{CO}$ and FT in Arabidopsis thaliana. Plant J 43:758-768. https:// doi.org/10.1111/j.1365-313X.2005.02491.x

Cho L-H, Pasriga R, Yoon J et al (2018) Roles of Sugars in Controlling Flowering Time. J Plant Biol 61:121-130. https://doi.org/10.1007/ s12374-018-0081-z

Coles ND, McMullen MD, Balint-Kurti PJ et al (2010) Genetic control of photoperiod sensitivity in maize revealed by joint multiple population ANALYSIS. Genetics 184:799-812. https://doi.org/ 10.1534/genetics.109.110304

Corbesier L, Vincent C, Jang S et al (2007) FT protein movement contributes to long-distance signaling in floral induction of Arabidopsis. Science 316(5827):1030-1033. https://doi.org/10.1126/ science. 1141752

Covarrubias-Pazaran G (2016) Genome-assisted prediction of quantitative traits using the $\mathrm{r}$ package sommer. PLoS ONE 11:e0156744

Emms DM, Kelly S (2015) OrthoFinder: solving fundamental biases in whole genome comparisons dramatically improves orthogroup inference accuracy. Genome Biol 16:157. https://doi.org/10.1186/ s13059-015-0721-2

Fornara F, de Montaigu A, Coupland G (2010) SnapShot: control of flowering in arabidopsis. Cell 141(550):550.e1-2. https://doi.org/ 10.1016/j.cell.2010.04.024

Fukuda M, Matsuo S, Kikuchi K et al (2011) Isolation and functional characterization of the FLOWERING LOCUS T homolog, the LsFT gene, in lettuce. J Plant Physiol 168:1602-1607. https://doi. org/10.1016/j.jplph.2011.02.004

Garrison E, Marth G (2012) Haplotype-based variant detection from short-read sequencing. arXiv: 12007.3907

Gendall AR, Levy YY, Wilson A, Dean C (2001) The VERNALIZATION 2 gene mediates the epigenetic regulation of vernalization in Arabidopsis. Cell 107:525-535

Han R, Truco MJ, Lavelle DO, Michelmore RW (2021a) A Composite analysis of flowering time regulation in lettuce. Front Plant Sci $12: 360$

Han R, Wong AJY, Tang Z et al (2021b) Drone phenotyping and machine learning enable discovery of loci regulating daily floral opening in lettuce. J Exp Bot 72:2979-2994. https://doi.org/10. 1093/jxb/erab081

Hartman Y, Hooftman DAP, Uwimana B et al (2012) Genomic regions in crop-wild hybrids of lettuce are affected differently in different environments: implications for crop breeding. Evol Appl 5:629640. https://doi.org/10.1111/j.1752-4571.2012.00240.x

Hartman Y, Hooftman DAP, Eric Schranz M, van Tienderen PH (2013a) QTL analysis reveals the genetic architecture of domestication traits in crisphead lettuce. Genet Resour Crop Evol 60:1487-1500. https://doi.org/10.1007/s10722-012-9937-0

Hartman Y, Uwimana B, Hooftman DAP et al (2013b) Genomic and environmental selection patterns in two distinct lettuce crop-wild hybrid crosses. Evol Appl 6:569-584. https://doi.org/10.1111/eva. 12043

Hassidim M, Harir Y, Yakir E et al (2009) Over-expression of CONSTANS-LIKE 5 can induce flowering in short-day grown arabidopsis. Planta 230:481-491. https://doi.org/10.1007/ s00425-009-0958-7

Higashi T, Aoki K, Nagano AJ et al (2016) Circadian oscillation of the lettuce transcriptome under constant light and light-dark conditions. Front Plant Sci 7:1114. https://doi.org/10.3389/fpls.2016. 01114

Imaizumi T, Schultz TF, Harmon FG et al (2005) FKF1 F-Box protein mediates cyclic degradation of a repressor of CONSTANS in Arabidopsis. Science 309(5732):293-297. https://doi.org/10. 1126/science. 1110586

Iñigo S, Alvarez MJ, Strasser B et al (2012) PFT1, the MED25 subunit of the plant Mediator complex, promotes flowering through CONSTANS dependent and independent mechanisms in Arabidopsis. Plant J 69:601-612. https://doi.org/10.1111/j.1365-313X.2011. 04815.x

Jang S, Marchal V, Panigrahi KCS et al (2008) Arabidopsis COP1 shapes the temporal pattern of $\mathrm{CO}$ accumulation conferring a photoperiodic flowering response. EMBO J 27:1277-1288

Jenni S, Truco MJ, Michelmore RW (2013) Quantitative trait loci associated with tipburn, heat stress-induced physiological disorders, and maturity traits in crisphead lettuce. Theor Appl Genet 126:3065-3079. https://doi.org/10.1007/s00122-013-2193-7

Johanson U, West J, Lister C et al (2000) Molecular analysis of FRIGIDA, a major determinant of natural variation in Arabidopsis flowering time. Science 290(5490):344-347. https://doi.org/ $10.1126 /$ science. 290.5490 .344

Kesseli R, Ochoa O, Michelmore R (1991) Variation at RFLP loci in Lactuca spp. and origin of cultivated lettuce (L. sativa). Genome 34:430-436. https://doi.org/10.1139/g91-065

Kwon S, Simko I, Hellier B et al (2013) Genome-wide association of 10 horticultural traits with expressed sequence tag-derived SNP markers in a collection of lettuce lines. Crop J. 1:25-33. https:// doi.org/10.1016/j.cj.2013.07.014

Lang A (1965). Physiology of flower initiation. In: Differenzierung und Entwicklung/Differentiation and Development. Springer. pp $1380-1536$.

Lavelle DO (2009) Genetics of Candidate Genes for Developmental and Domestication-Related Traits in Lettuce. University of California, Davis

Legris M, Ince YÇ, Fankhauser C (2019) Molecular mechanisms underlying phytochrome-controlled morphogenesis in plants. Nat Commun 10:5219. https://doi.org/10.1038/s41467-019-13045-0

Li H (2013) Aligning sequence reads, clone sequences and assembly contigs with BWA-MEM. arXiv:1303.3997

Li C, Gu M, Shi N et al (2011) Mobile FT mRNA contributes to the systemic florigen signalling in floral induction. Sci Rep 1:73. https://doi.org/10.1038/srep00073

Lim M-H, Kim J, Kim Y-S et al (2004) A new Arabidopsis gene, FLK, encodes an RNA binding protein with $\mathrm{K}$ homology motifs and regulates flowering time via FLOWERING LOCUS C. Plant Cell 16:731-740. https://doi.org/10.1105/tpc.019331 
Madeira F, Park YM, Lee J et al (2019) The EMBL-EBI search and sequence analysis tools APIs in 2019. Nucleic Acids Res 47:W636-W641. https://doi.org/10.1093/nar/gkz268

Maheswaran M, Huang N, Sreerangasamy SR, McCouch SR (2000) Mapping quantitative trait loci associated with days to flowering and photoperiod sensitivity in rice (Oryza sativa L.). Mol Breed 6:145-155. https://doi.org/10.1023/A:1009618621720

Mamo BE, Hayes RJ, Truco MJ et al (2019) The genetics of resistance to lettuce drop (Sclerotinia spp.) in lettuce in a recombinant inbred line population from Reine des Glaces $\times$ Eruption. Theor Appl Genet 132:2439-2460. https://doi.org/10.1007/ s00122-019-03365-6

Maynard L (2014) Cool season crops. https://ag.purdue.edu/hla/fruit veg/Presentations/maynard_cool-season-crops_ihc2014.pdf

Méndez-Vigo B, Martínez-Zapater JM, Alonso-Blanco C (2013) The Flowering Repressor SVP Underlies a Novel Arabidopsis thaliana QTL interacting with the genetic background. PLOS Genet 9:e1003289

Monte E, Alonso JM, Ecker JR et al (2003) Isolation and characterization of phyC mutants in Arabidopsis reveals complex crosstalk between phytochrome signaling pathways. Plant Cell 15:19621980. https://doi.org/10.1105/tpc.012971

Mouradov A, Cremer F, Coupland G (2002) Control of flowering time: interacting pathways as a basis for diversity. Plant Cell 14(Suppl):S111-S130. https://doi.org/10.1105/tpc.001362

Niroula M (2017) Environmental sensitivity of quantitative trait loci for seed germination and flowering time in lettuce (Lactuca sativa L.). University of California, Davis

Park DH, Somers DE, Kim YS et al (1999) Control of circadian rhythms and photoperiodic flowering by the Arabidopsis GIGANTEA gene. Science 285(5433):1579-1582. https://doi. org/10.1126/science.285.5433.1579

Putterill J, Robson F, Lee K et al (1995) The CONSTANS gene of arabidopsis promotes flowering and encodes a protein showing similarities to zinc finger transcription factors. Cell. https://doi. org/10.1016/0092-8674(95)90288-0

Rastas P (2017) Lep-MAP3: robust linkage mapping even for low-coverage whole genome sequencing data. Bioinformatics 33:37263732. https://doi.org/10.1093/bioinformatics/btx494

Reeves PA, He Y, Schmitz RJ et al (2007) Evolutionary conservation of the FLOWERING LOCUS C-mediated vernalization response: evidence from the sugar beet (Beta vulgaris). Genetics 176:295307. https://doi.org/10.1534/genetics.106.069336

Reyes-Chin-Wo S, Wang Z, Yang X et al (2017) Genome assembly with in vitro proximity ligation data and whole-genome triplication in lettuce. Nat Commun 8:14953. https://doi.org/10.1038/ ncomms 14953

Ryder EJ (1996) Ten Lettuce Genetic Stocks with Early Flowering Genes Ef-1ef-1 and Ef-2ef-2. HortScience HortSci. 31:473-475. https://doi.org/10.21273/HORTSCI.31.3.473

Sánchez-Lamas M, Lorenzo CD, Cerdán PD (2016) Bottom-up Assembly of the Phytochrome Network. PLOS Genet 12:e1006413

Sandoya G, Truco M-J, Bertier LD et al (2020) Genetics of partial resistance against verticillium dahliae race 2 in wild and cultivated Lettuce. Phytopathol. https://doi.org/10.1094/ PHYTO-09-20-0396-R

Searle I, Coupland G (2004) Induction of flowering by seasonal changes in photoperiod. EMBO J 23:1217-1222. https://doi.org/ 10.1038/sj.emboj.7600117
Searle I, He Y, Turck F et al (2006) The transcription factor FLC confers a flowering response to vernalization by repressing meristem competence and systemic signaling in arabidopsis. Genes Dev 20:898-912. https://doi.org/10.1101/gad.373506

Seki K, Komatsu K, Tanaka K et al (2020) A CIN-like TCP transcription factor (LsTCP4) having retrotransposon insertion associates with a shift from salinas type to Empire type in crisphead lettuce (Lactuca sativa L.). Hortic Res. 7:15. https://doi.org/10.1038/ s41438-020-0241-4

Song YH, Kubota A, Kwon MS et al (2018) Molecular basis of flowering under natural long-day conditions in Arabidopsis. Nat Plants 4:824-835. https://doi.org/10.1038/s41477-018-0253-3

Sourdille P, Snape JW, Cadalen T et al (2000) Detection of QTLs for heading time and photoperiod response in wheat using a doubledhaploid population. Genome 43:487-494. https://doi.org/10.1139/ g00-013

Srikanth A, Schmid M (2011) Regulation of flowering time: all roads lead to Rome. Cell Mol Life Sci 68:2013-2037. https://doi.org/ 10.1007/s00018-011-0673-y

Sthapit Kandel J, Peng H, Hayes RJ et al (2020) Genome-wide association mapping reveals loci for shelf life and developmental rate of lettuce. Theor Appl Genet 133:1947-1966. https://doi.org/10. 1007/s00122-020-03568-2

Suárez-López P, Wheatley K, Robson F et al (2001) CONSTANS mediates between the circadian clock and the control of flowering in Arabidopsis. Nature 410:1116-1120. https://doi.org/10.1038/ 35074138

Sukprakarn S (1985). A study of the effects of temperature and photoperiod on vegetative growth and seed production of leaf lettuce (Lactuca sativa L.) : a thesis presented in partial fulfilment of the requirement for the degree of Doctor of Philosophy in Seed Technology. Massey University

Tasma IM, Lorenzen LL, Green DE, Shoemaker RC (2001) Mapping genetic loci for flowering time, maturity, and photoperiod insensitivity in soybean. Mol Breed 8:25-35. https://doi.org/10.1023/A: 1011998116037

Thompson RC, Ryder EJ (1961). Descriptions and pedigrees of nine varieties of lettuce. US Department of Agriculture.

Wang K, Li M, Hakonarson H (2010) ANNOVAR: functional annotation of genetic variants from high-throughput sequencing data. Nucleic Acids Res 38:e164-e164. https://doi.org/10.1093/nar/ gkq603

Waycott W (1995) Photoperiodic response of genetically diverse lettuce accessions. J Am Soc Hortic Sci 120(460):467. https://doi.org/10. 21273/JASHS.120.3.460

Wimmer V, Albrecht T, Auinger H-J, Schön C-C (2012) synbreed: a framework for the analysis of genomic prediction data using R. Bioinformatics 28:2086-2087. https://doi.org/10.1093/bioin formatics/bts335

Zhang L, Su W, Tao R et al (2017) RNA sequencing provides insights into the evolution of lettuce and the regulation of flavonoid biosynthesis. Nat Commun 8:2264. https://doi.org/10.1038/ s41467-017-02445-9

Publisher's Note Springer Nature remains neutral with regard to jurisdictional claims in published maps and institutional affiliations. 